\title{
Prediction of Early Rebleeding and Mortality after Acute Esophageal Variceal Hemorrhage among Yemeni Patients in Major Hospitals-Sana’a
}

\author{
Arege Yahya Hunaysh \\ Department of Internal Disease and Gastroenterology and Endoscopy Unit, Sana'a University, Sana'a, Yemen \\ Email: Arege.Hunaysh@yahoo.com
}

Received 19 March 2016; accepted 20 August 2016; published 23 August 2016

Copyright (C) 2016 by author and Scientific Research Publishing Inc.

This work is licensed under the Creative Commons Attribution International License (CC BY). http://creativecommons.org/licenses/by/4.0/

(c) (i) Open Access

\section{Abstract}

Background and aims: Acute variceal hemorrhage (AVH) is the most serious encountered complication of liver cirrhosis and carries high mortality rate. Several risk factors that predict early rebleeding and mortality have been studied and there is no similar study in our country, so the aim of this study was to identify the risk factors of early rebleeding and mortality after bleeding episode in cirrhotic patients in Yemen. Patients and Method: It was a prospective study of cirrhotic patients with AVH who were admitted to the main public hospitals in Sana'a between April 2014 and March 2015. Demographic information, medical histories, physical examination findings, and laboratory test results were collected. Endoscopic and pharmacologic treatment was performed. The patients were followed up since admission and up to 6 weeks for the occurrence of rebleeding and mortality after the acute attack. Univariate and multivariate analyses were performed to identify independent risk factors for rebleeding and mortality. Survival analysis was estimated using the Kaplan-Meier method. Result: A total of 102 patients were analyzed. 26 patients $(25.5 \%)$ rebleeded within 6 weeks period. The predictive factors significantly associated with rebleeding within 6 weeks period in univariate analysis were clot on varix at index endo- scope $(P<0.0001)$, high serum bilirubin $(P=0.02)$, CTP score $(P<0.05)$ and MELD score $(P<0.05)$. Independent risks factors remained in multivariate analysis as significant predictors of early rebleeding were clot on varix (OR: 11.6, CI: 2.87 - 47.29, $P=0.001$ ) and high serum bilirubin (>3) (OR: 1.1, CI: 1.02 - 1.19, $P$ $=0.01)$. Sixteen patients died $(15.7 \%)$ within 6 weeks period. Predictors of mortality with significant difference in univariate analysis were hypovolemic shock $(P=0.001)$, high WBCs count $(P<0.0001)$, low serum sodium $(P=0.04)$, high AST $(P<0.0001)$, high ALT $(P=0.02)$, high INR ( $P$ $<0.0001)$, high serum bilirubin $(P<0.0001)$, low serum albumin $(P=0.005)$, ascites $(P=0.001)$, 
CTP score $(\mathrm{P}<0.0001)$ and MELD score $(\mathrm{P}<\mathbf{0 . 0 0 0 1})$. By multiple regression analysis high MELD score ( $\geq 19$ ) (OR: 1.3, CI: 1.12 - 1.41, P < 0.0001) and WBCs over 10.3 × 10\%/l (OR: 1.2, CI: 1.02 - 1.40, $P<0.05)$ were independent risk factors for mortality within 6 weeks period. Conclusion: Early rebleeding in cirrhotic patients with AVH was associated with clot on varix at endoscope and high serum bilirubin more than $3 \mathrm{mg} / \mathrm{dl}$. Early mortality rate was associated with high MELD score $(\geq 19)$ and WBCs over $10.3 \times 10^{9} / 1$.

\title{
Keywords
}

\author{
Acute Variceal Hemorrhage, Child-Turcotte-Pugh Score, Model for End-Stage Liver Disease \\ (MELD), Prospective Study, Univariate Analysis, Multivariate Analysis
}

\section{Introduction}

Variceal bleeding is a serious complication of liver cirrhosis and is associated with significant morbidity and mortality [1] [2]. It causes $70 \%$ of all upper gastrointestinal bleeding [3]. Approximately $50 \%$ of patients with cirrhosis have esophageal varices. Bleeding from varices occurs in $30 \%$ of patients. The risk of recurrent episodes of variceal hemorrhage increases after the first esophageal variceal hemorrhage [3]. There are several factors that may increase the risk of bleeding. The most important are: the size of the varices, the presence at endoscopy of red wale markings, the severity of liver disease and active alcohol abuse [4]. Also there are several factors that have been identified as predictors of mortality after esophageal variceal hemorrhage, including early rebleeding, bacterial infection, hepatic venous pressure gradient (HVPG) $>20 \mathrm{mmHg}$ measured shortly after admission, active bleeding at initial endoscopy, severity of initial bleeding, hematocrit level, transaminases levels, presence of portal vein thrombosis, hepatocellular carcinoma (HCC), alcoholic liver disease, serum bilirubin and albumin levels. Other predictors of mortality are Child-Turcotte-Pugh (CTP) score (class B and C). Historically Child-Turcotte-Pugh score is used to stratify patients at risk for death after episode of acute variceal hemorrhage (AVH). CTP classification is a widely used index of disease severity for patient with end stage liver disease. Recently Model for End-stage Liver Disease (MELD) score was developed to predict mortality of patient with cirrhosis [5]-[7]. As we noted, several risk factors that predict early rebleeding and mortality have been studied and there is no similar study in our country, so the aim of this study was to identify the risk factors of early rebleeding and mortality after bleeding episode in cirrhotic patients in Yemen.

\section{Patients and Method}

A prospective study of cirrhotic patients who presented with episodes of acute variceal bleeding admitted to the main public hospitals in Sana'a, Yemen (Al-Kuwait university hospital, Al-Thawra Hospital and Al-Gumhory Teaching Hospital) was conducted between April 2014 and March 2015. Liver cirrhosis diagnosis was based on a combination of physical sings, biochemical (low serum albumin, elevated serum bilirubin or prothrombin time prolongation) and imaging data (ultrasound and computed tomography). Esophageal variceal bleeding would be confirmed by endoscopy. Patients with hepatocellular carcinoma or other neoplasm, bleeding from gastrointestinal tract due to causes other than esophageal varices, without complete data, lost follow up 6 weeks period, received management of variceal hemorrhage with somostatin and somostatin analogues within the previous 12 hours would be exclude from the study. Before collecting the data from the patients the following definition criteria were used [2] [8]-[10]. Active bleeding: persistent bleeding at endoscope, visible oozing or spurting of blood from a varix. Stigmata of recent bleeding or high risk stigmata: the presence of an adherent clot, white nipple or red signs on varices (cherry red spot, red wale sign or haematocystic spots); or the presence of varices and blood in the stomach; or the presence of large varices in the setting of a patient presenting with haematemesis. Early rebleeding: recurrence of clinically significant hemorrhage (hematemesis/melena, aspiration of greater than $100 \mathrm{~mL}$ of fresh blood from nasogastric tube or $>3 \mathrm{~g} / \mathrm{dL}$ decrease of $\mathrm{Hb}$ if no transfusion is given) within 6 weeks after index bleeding episode was considered finished. Early mortality: death occurring within 6 weeks after bleeding. Arterial hypotension: reduction in mean arterial pressure of $25 \%$ or greater with respect to baseline values with a final value of $70 \mathrm{mmHg}$. Infection was diagnosed during the first 5 days after the index hemorr- 
hage if fever greater than $37.5^{\circ} \mathrm{C}$ for more than 12 hours or compatible clinical, X-ray, or positive results of blood, sputum, urine, and ascites bacterial culture or elevated ascites fluid and absolute neutrophil count (ANC) $\geq 250$ cells $/ \mu \mathrm{L}$ were present. The presence of fever $>38^{\circ} \mathrm{C}$ that lasted more than 24 hours and left shift of elevated WBCs and no any other evidence of infection is highly suggestive of possible infections [11]. Data were collected from the patients at the time of admission (designated as time zero) by using a structured protocol /questionnaire. The questionnaire designed for proposing complete assessment of the patients which include demographic data, medical history, physical examination with vital signs, causes of liver diseases, laboratory date (complete blood count, serum creatinine, total bilirubin, serum albumin, prothrombin time, international normalized ratio (INR), aspartate and alanine aminotransferase levels and serum sodium). CTP classification and MELD score calculation were evaluated. Endoscope was performed by experienced endoscopist. All patients with upper gastrointestinal bleeding underwent endoscopic examination within 24 hours of presentation to identify the source of bleeding. Endoscopic finding were documented, including the grade of varix, the presence of stigmata of recent bleeding, high risk stigmata or active bleeding. At the time of admission all patients were managed by fluid resuscitation to maintain systolic blood pressure of $90-100 \mathrm{mmHg}$ and pulse rate of 100 beats per minute. Patients with a significant drop in hemoglobin level $(<8 \mathrm{~g} / \mathrm{dl})$ were given packed red blood cell (PRBC) transfusions to maintain hemoglobin level between $8 \mathrm{~g} / \mathrm{dl}-10 \mathrm{~g} / \mathrm{dl} .2$ - 5 days course of octreotide infusion $(50 \mu \mathrm{g}$ i.v. bolus followed by a continuous infusion of $25-50 \mu \mathrm{g} / \mathrm{h})$ was performed. Patients with cirrhosis and upper gastrointestinal bleeding are at high risk of bacterial infection so 5 days course of antibiotic prophylaxis were managed (ceftriaxone i.v. $1 \mathrm{~g} / 12 \mathrm{~h}$ ). Secondary prophylaxis with propranolol (80 - $160 \mathrm{mg}$ daily) was prescribed. MELD score was calculated according to the formula $\left(9.57 \times \log _{\mathrm{e}}\right.$ (creatinine) $+3.78 \times \log _{\mathrm{e}}$ (total bilirubin) +11.2 $\times \log _{e}(\mathrm{INR})+6.43$.) which was obtained from on-line worksheet (WWW.hepatitisc.uw.edu/go/managment) The severity of cirrhosis was graded according to Pugh's modification of Child's scoring. Then patients were followed for early rebleeding and mortality for 6 weeks (end point). Patients with rebleeding within 6 weeks were classified as rebleeding group, the remaining patients were considered as control group. Patient's survival would assess as the interval from the day of admission until death or last follow up. Patients who died were classified as mortality group and survived patients were considered as survival group.

\section{Results}

Between April 2014 and March 2015, 160 cirrhotic patients with features of acute upper gastrointestinal bleeding suspicious for variceal hemorrhage were enrolled in the study at the major hospitals in Sana'a (77 patients in Al-Kuwait university hospital, 62 patients in Al-Thwrah Hospital and 21 patients in Al-Gumhory Teaching Hospital). 58 patients were excluded from the analysis because of $\mathrm{HCC}(\mathrm{n}=9)$ and inability to follow them $(\mathrm{n}=$ 49). Therefore, the number of patients who met the inclusion criteria and were analyzed in the current study was 102. Baseline demographic, etiology of cirrhosis, clinical and biochemical characteristics of the patients at admission are presented in Table 1. The median (IQR) age of the patients were 40 (30 - 59) with 79 (77.5\%) men and $23(22.5 \%)$ women. The most frequent etiology of liver cirrhosis was viral hepatitis in $58(56.8 \%)$ patients (33 patients had hepatitis B and 25 had hepatitis C) followed by autoimmune hepatitis in 33 (32.4\%) patients and Bilharzias in $11(10.8 \%)$ patients. Nine point eight percent of patients had diabetes mellitus and two point nine percent of patients had hypertension. Thirty six patients were hemodynamically unstable at admission and 66 patients were hemodynamically stable. About $47 \%$ of patients presented with moderate to severe ascites. Twenty six patients had hepatic encephalopathy. Hepatic encephalopathy grade I/II occurred in $46.1 \%$ of patients and $53.9 \%$ of patients had grade III/IIII. The mean hemoglobin level was $7.9 \pm 2.3$. The median white blood cell was $5.8(2.9$ - 9.3). The median platelet count was 89.5 (59.5 - 131). The mean serum albumin was $2.8 \pm 0.6$. The mean serum sodium was $135.1 \pm 4.8$. The median serum bilirubin was $1.5(0.9-5.5)$. The median serum creatinine was $0.8(0.6$ - 1). The median INR was $1.3(1.2-1.8)$. Infection occurred in $12(11.8 \%)$ patients. Median CTP and MELD score at the time of admission were 8 ( 7 - 10) and 13.3 (9.7 - 19.3), respectively. $79.4 \%$ of patients had CTP class B/C. All patients underwent emergency endoscopy. Endoscopic findings are summarized in Table 2. The majority of patients (69.6\%) had esophageal varices alone, and $30.4 \%$ had esophageal varices associated with gastric varices. Esophageal varices grade II, III, and IV were observed in $14.8 \%$, 58.7 and $26.5 \%$ of patients respectively. Sixteen $(15.7 \%)$ patients had active bleeding during the procedure. Stigma of recent bleeding in form of clot on varix was found in 56 (54.9\%) patients. High risk stigmata were found in $76(75.6 \%)$ patients. Bands were applied to 73 patients and 29 patients received sclerotherapy. 
Table 1. Demographic Clinical and Biochemical characteristics of the patient at admission $(\mathrm{n}=102)$.

\begin{tabular}{|c|c|}
\hline \multicolumn{2}{|l|}{ Variable } \\
\hline Age (years) ${ }^{*}$ & $40(30-59)$ \\
\hline $\begin{array}{c}\text { Gender } \\
\text { Male n (\%) } \\
\text { Female n (\%) } \\
\text { Etiology }\end{array}$ & $\begin{array}{l}79(77.5) \\
23(22.5)\end{array}$ \\
\hline Viral n $(\%)$ & $58(56.9)$ \\
\hline Non-viral n (\%) & $44(43.1)$ \\
\hline Comorbidity n (\%) & $15(14.7)$ \\
\hline $\begin{array}{c}\text { Ascites n (\%) } \\
\text { None/mild } \\
\text { Moderate/severe }\end{array}$ & $\begin{array}{l}55(54) \\
47(46)\end{array}$ \\
\hline Hypovolemic shock & $36(35.3)$ \\
\hline Encephalopathy n (\%) & $26(25.5)$ \\
\hline Active bleeding n (\%) & $16(15.7)$ \\
\hline Stigmata of recent bleeding $\mathrm{n}(\%)$ & $56(54.9)$ \\
\hline Portal hypertension n (\%) & $93(91.2)$ \\
\hline Esophageal varices & $61(59.8)$ \\
\hline $\mathrm{Hb}($ mean $\pm \mathrm{SD})$ & $7.9 \pm 2.3$ \\
\hline $\mathrm{WBC}^{*}$ & $5.8(2.9-9.3)$ \\
\hline Platelets $^{*}$ & $89.5(59.5-131)$ \\
\hline Serum Albumin & $2.8 \pm 0.6$ \\
\hline Serum $\mathrm{Na}$ & $135.1 \pm 4.8$ \\
\hline Serum bilirubin ${ }^{*}$ & $1.5(0.8-5.1)$ \\
\hline $\mathrm{ALT}^{*}$ & $38.8(22.2-64.3)$ \\
\hline AST $^{*}$ & $47.5(31-79.8)$ \\
\hline Serum creatinine ${ }^{*}$ & $0.8(0.6-1)$ \\
\hline $\mathrm{INR}^{*}$ & $1.3(1.2-1.8)$ \\
\hline Fever & $12(11.8)$ \\
\hline CPT score* & $8(7-10)$ \\
\hline CPT class $(\%)$ & \\
\hline A & $21(20.6)$ \\
\hline B & $50(49)$ \\
\hline $\mathrm{C}$ & $31(30.4)$ \\
\hline MELD score* & $13.3(9.7-19.3)$ \\
\hline Systolic blood pressure ${ }^{*}$ & $90(80-100)$ \\
\hline
\end{tabular}

*Median (IQR).

Table 2. Endoscopy findings of patients $(n=102)$.

\begin{tabular}{cc}
\hline Variables & $\mathrm{N}(\%)$ \\
\hline Esophageal varices alone & $69.6 \%$ \\
Esophageal varices associated with gastric & $30.4 \%$ \\
varices & \\
Esophageal varices grade & $14.8 \%$ \\
$\bullet$ II & $58.7 \%$ \\
$\bullet$ II & $26.5 \%$ \\
Active bleeding & $16(15.7)$ \\
Clot on varix & $56(54.9)$ \\
High risk stigmata & $76(75.6)$ \\
\hline
\end{tabular}




\section{Outcome}

Among 102 cirrhotic patients with acute variceal hemorrhage, rebleeding episodes within 5 days occurred in $14.7 \%$. Twenty six patients (25.5\%) suffered rebleeding within 6 weeks period and more than $50 \%$ of these patients rebleed within 5 days. Cumulative probability of 6 weeks rebleeding (remaining free of variceal rebleeding) after acute variceal hemorrhage, is shown in Figure 1. Ten variables related to clinical and ten biochemical data in rebleeding and non-rebleeding group were compared. The clinical findings and laboratory data that predict early rebleeding in univariate analysis are shown in Table 3 . The predictive factors significantly associated with rebleeding within 6 weeks period in univariate analysis were clot on varix at index endoscope $(\mathrm{P}<0.0001)$, serum bilirubin $(\mathrm{P}=0.02)$, CTP $(\mathrm{P}<0.05)$ and MELD score $(\mathrm{P}<0.05)$. Independent risk factors remained in multivariate analysis (Table 4) as significant predictors of early rebleeding were clot on varix (odd ratio (OR): 11.06, Confidence interval (CI): $2.87-47.29, \mathrm{P}<0.005$ ) and serum bilirubin (OR: 1.1, CI: $1.02-1.19, \mathrm{P}<$ 0.05). Bilirubin level of more than $3 \mathrm{mg} / \mathrm{dl}$ was associated with Area Under the Receiver Operating Characteristics (AUROC) of 0.65, P < 0.05, CI: $0.53-0.78$ (Figure 2). Cumulative probability of 6 weeks rebleeding (remaining free of variceal rebleeding) stratified according to serum bilirubin less than or equal to $3 \mathrm{mg} / \mathrm{dl}$ and more than $3 \mathrm{mg} / \mathrm{dl}$ are presented in Figure 3 .

Sixteen patients died (15.7\%) within 6 weeks period. Mortality rate in 5 days period was $11.8 \%$. Kaplan-Meier plots showing the cumulative probability of 6 weeks survival for 102 patients included in the study (Figure 4). The clinical findings of both 6 weeks mortality group and 6 weeks survival group are compared and presented in Table 5. Predictors of mortality with significant difference ( $\mathrm{P}$ value of $<0.0001$ ) in univariate analysis were WBC count, AST, INR, bilirubin, CTP score and MELD score. Ascites $(\mathrm{P}=0.001)$, hypovolemic shock $(\mathrm{P}<0.001)$, albumin $(\mathrm{P}=0.005)$, ALT $(\mathrm{P}<0.02)$ and serum sodium $(\mathrm{P}=0.04)$ also were differently significant between two group. By multiple regression analysis high MELD score (OR: 1.3, CI: 1.12 - 1.41, P < $0.0001)$ and high WBCs count (OR: $1.2, \mathrm{CI}: 1.02-1.40, \mathrm{P}<0.05)$ were an independent risk factors for mortality within 6 weeks period (Table 6).

The cut off values for MELD score in predicting six weeks mortality, that maximize specificity and optimize sensitivity were constructed using ROC. Optimized cut-off value of 19 points for equally important sensitivity

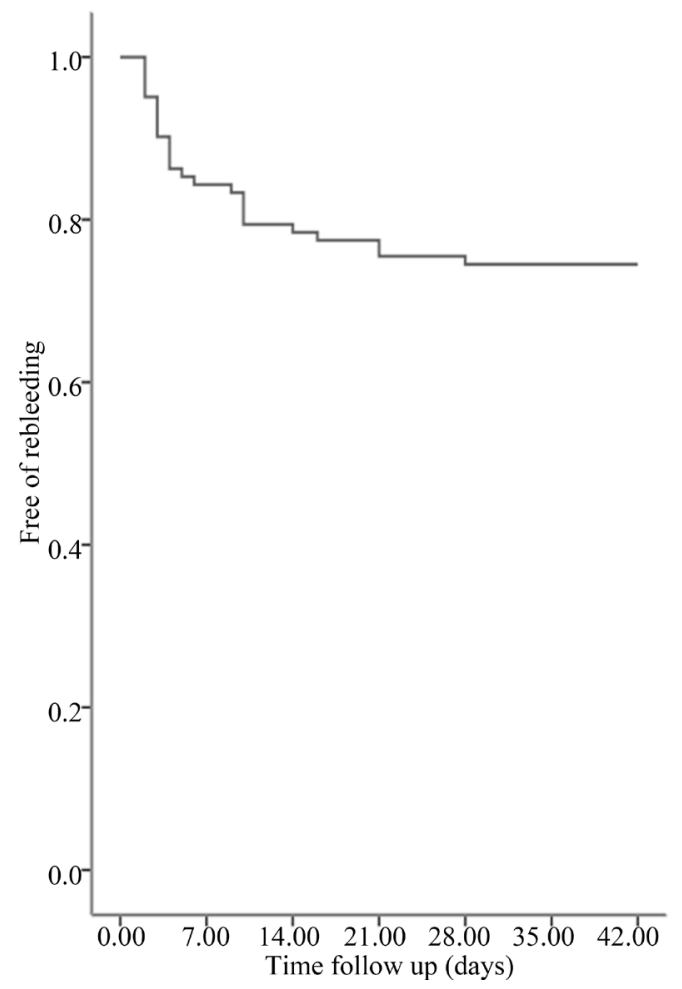

Figure 1. Kaplan-Meier estimates of cumulative probability of 6 weeks rebleeding (remaining free of variceal rebleeding) after acute variceal hemorrhage. 
Table 3. Comparison of relevant variable predicting rebleeding within 6-week after acute vacireal hemorrhage.

\begin{tabular}{|c|c|c|c|c|c|}
\hline Variable & $\begin{array}{l}\text { Non-rebleeding } \\
(\mathrm{N}=76,74.5 \%)\end{array}$ & $\begin{array}{c}\text { Rebleeding } \\
(\mathrm{N}=26,25.5 \%)\end{array}$ & OR & $95 \% \mathrm{CI}$ & P Value \\
\hline Age (years) $)^{*}$ & $40(28-60)$ & $50(35-59)$ & & & $P=0.53$ \\
\hline $\begin{array}{c}\text { Gender n (\%) } \\
\text { Male } \\
\text { Female }\end{array}$ & $\begin{array}{l}68(79.1) \\
18(20.9)\end{array}$ & $\begin{array}{l}11(68.8) \\
5(31.2)\end{array}$ & 0.85 & $0.18-1.96$ & $\mathrm{P}=0.35$ \\
\hline $\begin{array}{c}\text { Etiology n (\%) } \\
\text { Viral } \\
\text { Non-viral }\end{array}$ & $\begin{array}{l}42(55.3) \\
34(44.7)\end{array}$ & $\begin{array}{c}16(61.5) \\
10(38.5)\end{array}$ & 1.30 & $0.52-3.22$ & $\mathrm{P}=0.74$ \\
\hline $\begin{array}{c}\text { Comorbidity n (\%) } \\
\text { Yes } \\
\text { No }\end{array}$ & $\begin{array}{ll}11 & (14.5) \\
65 & (85.5)\end{array}$ & $\begin{array}{c}4(15.4) \\
22(84.6)\end{array}$ & 1.07 & $0.31-3.72$ & $\mathrm{P}=1$ \\
\hline $\begin{array}{c}\text { Hypovolemic shock n (\%) } \\
\text { Present } \\
\text { Absent }\end{array}$ & $\begin{array}{l}23(30.3) \\
53(69.7)\end{array}$ & $\begin{array}{l}13(50) \\
13(50)\end{array}$ & 2.30 & $0.90-5.70$ & $\mathrm{P}=0.1$ \\
\hline $\begin{array}{c}\text { Ascites n (\%) } \\
\text { None/mild } \\
\text { Moderate/severe }\end{array}$ & $\begin{array}{l}43(56.6) \\
33(43.4)\end{array}$ & $\begin{array}{l}12(46.2) \\
14(53.8)\end{array}$ & 1.5 & $0.6-3.7$ & $\mathrm{P}=0.5$ \\
\hline $\begin{array}{c}\text { Encephalopathy n (\%) } \\
\text { Present } \\
\text { Absent }\end{array}$ & $\begin{array}{l}16(21.1) \\
60(78.9)\end{array}$ & $\begin{array}{l}10(38.5) \\
16(61.5)\end{array}$ & 2.45 & $0.98-6.12$ & $\mathrm{P}=0.09$ \\
\hline Active bleeding $\mathrm{n}(\%)$ & $11(14.5)$ & $5(19.2)$ & 1.4 & $0.44-4.5$ & $\mathrm{P}=0.6$ \\
\hline $\begin{array}{c}\text { Clot on varix at endoscope } \\
\mathrm{n}(\%)\end{array}$ & $33(43.4)$ & $23(88.5)$ & 10 & $2.76-36.14$ & $\mathrm{P}<0.0001$ \\
\hline $\mathrm{Hb}($ mean $\pm \mathrm{SD})$ & $7.7 \pm 2.2$ & $8.8 \pm 2.2$ & & & $\mathrm{P}=0.07$ \\
\hline $\mathrm{WBC}^{*}$ & $6(2.8-9.3)$ & $5.6(4.1-5.6)$ & & $0.8-5.2$ & $\mathrm{P}=0.7$ \\
\hline Platelets ${ }^{*}$ & $90(55.8-150.3)$ & $87.8(72.8-115.3)$ & & & $\mathrm{P}=0.8$ \\
\hline Albumin ${ }^{*}$ & $2.7(2.3-3.1)$ & $2.7(2.2-3.1)$ & & & $\mathrm{P}=0.7$ \\
\hline Bilirubin $^{*}$ & $1.3(0.8-2.8)$ & $3.5(1.1-7.2)$ & & & $\mathrm{P}=0.02$ \\
\hline $\mathrm{ALT}^{*}$ & $41.3(24-70)$ & $30.8(19-56.8)$ & & & $\mathrm{P}=0.2$ \\
\hline AST $^{*}$ & $44.8(31.5-78.5)$ & $54(28.5-87.8)$ & & & $\mathrm{P}=0.9$ \\
\hline Serum creatinine ${ }^{*}$ & $0.8(0.6-1.1)$ & $0.8(0.7-0.9)$ & & & $\mathrm{P}=0.7$ \\
\hline Serum $\mathrm{Na}^{*}$ & $136(132.3-139)$ & $134(131.3-139.3)$ & & & $\mathrm{P}=0.2$ \\
\hline $\mathrm{INR}^{*}$ & $1.3(1.2-1.6)$ & $1.8(1.1-2.5)$ & & & $\mathrm{P}=0.4$ \\
\hline CPT score & $8(7-9)$ & $9.5(7-13)$ & & & $\mathrm{P}<0.05$ \\
\hline $\begin{array}{l}\text { CPT class } \\
\text { A } \\
\text { B } \\
\text { C }\end{array}$ & $\begin{array}{cc}7 & (22.4) \\
41 & (53.9) \\
18 & (23.7)\end{array}$ & $\begin{array}{l}4(15.4) \\
9(34.6) \\
13(50)\end{array}$ & & & $\mathrm{P}=0.04$ \\
\hline MELD score ${ }^{*}$ & $12.5(9.3-17.8)$ & $17.3(10-23.7)$ & & & $\mathrm{P}<0.05$ \\
\hline Systolic blood pressure $^{*}$ & $90(80-100)$ & $85(80-100)$ & & & $\mathrm{P}=0.2$ \\
\hline
\end{tabular}

*Median (IQR).

Table 4. Multivariate analysis of independent predictors for 6-week rebleeding after acute variceal hemorrhage.

\begin{tabular}{cccccrr}
\hline Variables & B estimate & S.E & Wald & OR & P value & 0.001 \\
Clot on varix & 2.45 & 0.716 & 11.73 & 11.6 & $0.87-47.29$ \\
Bilirubin & 0.095 & 0.038 & 6.16 & 1.1 & 0.01 & $1.02-1.19$ \\
\hline
\end{tabular}




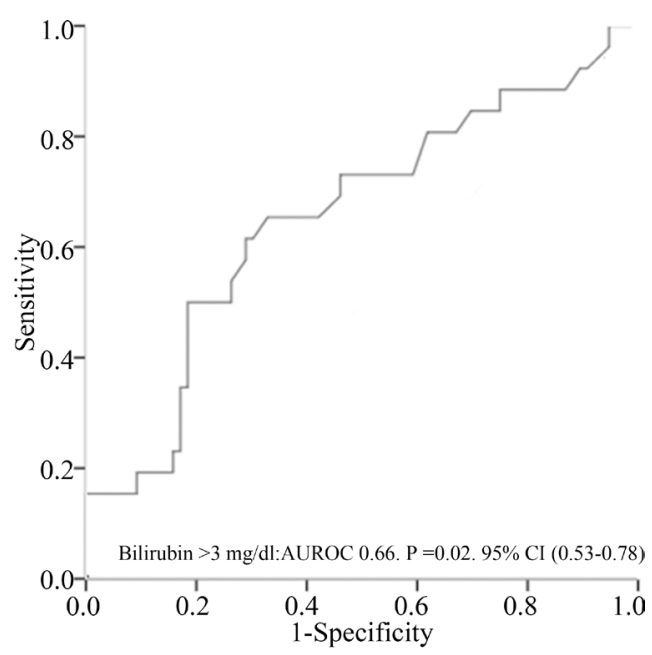

Figure 2. Receiver operating characteristic curve of cumulative probability of 6 week rebleeding (remaining free of variceal rebleeding) after acute variceal hemorrhage based in serum bilirubin $>3$ $\mathrm{mg} / \mathrm{dll}$.

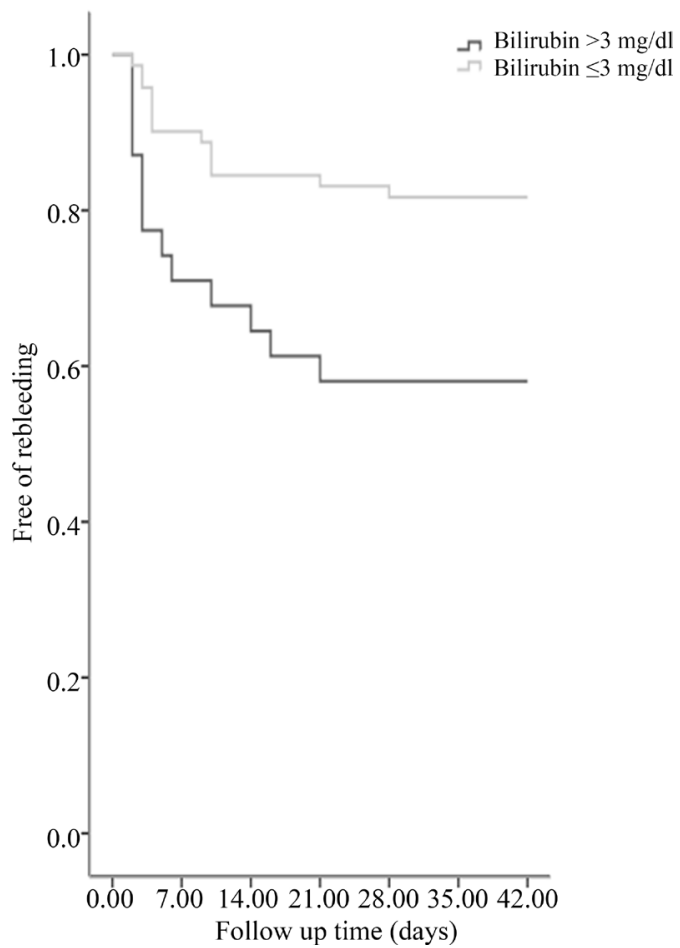

Figure 3. Kaplan-Meier plot of cumulative probability of 6 weeks rebleeding (remaining free of variceal rebleeding) after acute variceal hemorrhage based in serum bilirubin $>3 \mathrm{mg} / \mathrm{dl}$.

and specificity was chosen as a useful threshold to identify the patients with high risk of death within 6 weeks. The current study found that MELD score of 19 point had the best sensitivity and specificity for predicting 6 weeks mortality after acute variceal hemorrhage. A MELD score of $\geq 19$ was associated with Area Under the Receiver Operating Characteristics (AUROC) of 0.84, P $<0.001$, CI: $0.70-0.98$ (Figure 5). Stratification of the patients according to baseline MELD score above or below 19 (high MELD score $\geq 19$ and low MELD score $<19)$ revealed a significant difference in 6 week survival between patients with MELD score of $\geq 19$ compared to MELD score $<19(\mathrm{P}<0.001)$ (Figure 6). WBCs count of more than $10.3 \times 10^{9} / 1$ was associated with Area Under the Receiver Operating Characteristics (AUROC) of 0.78, $\mathrm{P}>0.0001$ (Figure 7). 


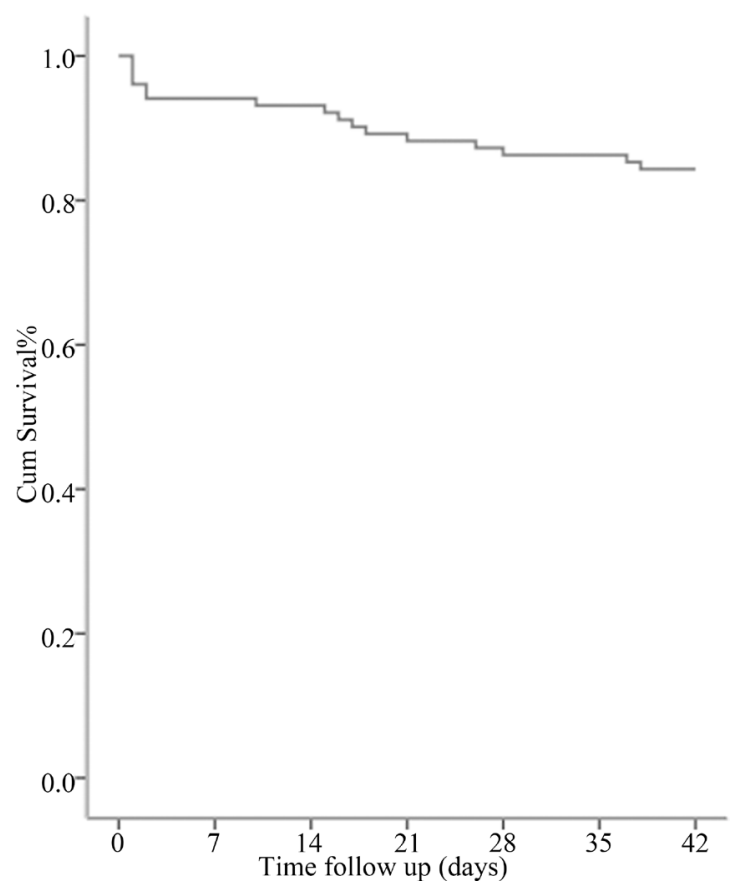

Figure 4. Kaplan-Meier survival curve after acute variceal hemorrhage.

\section{Discussions}

Acute variceal hemorrhage is the most serious encountered complication of liver cirrhosis and carries high mortality rate. Early rebleeding and mortality rate increase sharply within 6 weeks period then stabilized. Early rebleeding rate in the current study was $25.5 \%$, a similar result reported by Krige et al. [12] but it is slightly higher than that in some other studies [3] [13] while it is lower than that in other studies [14] [15].

The present study shows that several risk factors were associated with early rebleeding in univariate analysis but only few variables remained in multivariate analysis with significant difference between groups were identified as prognostic indicators of rebleeding within 6 weeks period. In univariate analysis clot on varix, serum bilirubin, CTP score and MELD score were predictive indicators of rebleeding within 6 weeks. Independent risk factors that predicts early rebleeding in multivariate analysis were clot on varix $(\mathrm{P}<0.0001, \mathrm{OR}=11.6,95 \%$ CI: 2.87 - 47.29) and serum bilirubin ( $<<0.05, \mathrm{OR}=1.1,95 \% \mathrm{CI}$ : $1.02-1.19)$, which was consistent with other studies [3] [12] [13] [16] [17]. D'Amico et al. [18], demonstrated that active bleeding at endoscopy is only independent predictor of early rebleeding, but not of death, while in our study clot on varix was independent predictor of early rebleeding.

Our study found that 6 weeks mortality rate were $15.7 \%$, which was comparable with the findings of other series [19]-[21]. Other studies found that 10 days and 2 weeks mortality rate were $11.5 \%$ [15] and $8 \%$ [22] respectively. Univariate analysis of clinical variables in this study showed that, hypovolemic shock, ascites, WBCs count, serum bilirubin, serum albumin, ALT, AST, CTP score and MELD score were risk factors associated with 6-week mortality. A subsequent, multivariate analysis stated that only MELD score $(\geq 19)$ and initial WBC over $10.3 \times 10^{9} / 1$ were significantly predicts mortality within 6 weeks.

MELD score based on objective variable available at admission were analyzed in several studies to predict 6 weeks mortality after acute variceal hemorrhage in patients with cirrhosis. Additionally, recent studies on the performance of MELD model observed that MELD objectively and accurately predicts mortality after acute variceal hemorrhage and MELD score were superior to child score in predicting 6 weeks mortality. Semper et al. [23] show that Child score of $\geq 10$ and MELD score of $\geq 18$ were independent predictors of mortality at 6 weeks and MELD score was superior to child score in predicting 6 weeks mortality. Chen et al. [24] described that MELD score was an easy and powerful predictor for 6-wk mortality and outcomes of patients with early rebleeding after EVL for esophageal variceal hemorrhage. Hunter et al. [16] confirmed that MELD score (>18), presence of active bleeding or white nipple sign at time of endoscopy were significant predictors for early re- 
Table 5. Comparison of relevant variable predicting 6-week mortality after acute vacireal hemorrhage.

\begin{tabular}{|c|c|c|c|c|c|}
\hline Variable & $\begin{array}{l}6 \text { week survival group } \\
(\mathrm{N}=86,84.3 \%)\end{array}$ & $\begin{array}{c}6 \text { week mortality } \\
\text { group } \\
(\mathrm{N}=16,15.7 \%)\end{array}$ & OR & $95 \% \mathrm{CI}$ & P Value \\
\hline Age (years) ${ }^{*}$ & $40(30-57)$ & $50(26-60)$ & & & $\mathrm{P}=0.8$ \\
\hline $\begin{array}{c}\text { Gender n (\%) } \\
\text { Male } \\
\text { Female } \\
\text { Etiology n (\%) }\end{array}$ & $\begin{array}{l}68(79.1) \\
18(20.9)\end{array}$ & $\begin{array}{ll}11 & (68.8) \\
5 & (31.2)\end{array}$ & 0.85 & $0.18-1.96$ & $\mathrm{P}=0.4$ \\
\hline $\begin{array}{c}\text { Viral } \\
\text { Non-viral }\end{array}$ & $\begin{array}{l}49(57) \\
37(43)\end{array}$ & $\begin{array}{l}9(56.2) \\
7(43.8)\end{array}$ & 0.97 & $0.33-2.85$ & $P=1$ \\
\hline $\begin{array}{c}\text { Comorbidity n }(\%) \\
\text { Yes } \\
\text { No }\end{array}$ & $\begin{array}{l}12(14) \\
74(86)\end{array}$ & $\begin{array}{c}3(18.8) \\
13(81.2)\end{array}$ & & & $\mathrm{P}=0.7$ \\
\hline $\begin{array}{c}\text { Hypovolemic shock n (\%) } \\
\text { Present } \\
\text { Absent } \\
\text { Ascites n (\%) }\end{array}$ & $\begin{array}{l}24(27.9) \\
62(72.1)\end{array}$ & $\begin{array}{l}12(75) \\
4(25)\end{array}$ & 7.75 & $2.28-26.40$ & $\mathrm{P}=0.001$ \\
\hline $\begin{array}{c}\text { None/mild } \\
\text { Moderate/severe } \\
\text { Encephalopathy n (\%) }\end{array}$ & $\begin{array}{ll}53 & (61.6) \\
33 & (38.4)\end{array}$ & $\begin{array}{c}2(12.5) \\
14(87.5)\end{array}$ & 11.24 & $2.4-52.7$ & $\mathrm{P}=0.001$ \\
\hline $\begin{array}{l}\text { Present } \\
\text { Absent }\end{array}$ & $\begin{array}{l}19(22.1) \\
67(77.9)\end{array}$ & $\begin{array}{ll}7(43.7) \\
9(56.3)\end{array}$ & 2.7 & $0.9-8.3$ & $\mathrm{P}=0.1$ \\
\hline Active bleeding $\mathrm{n}(\%)$ & $12(14)$ & $4(25)$ & 2.06 & $0.6-7.4$ & $\mathrm{P}=0.3$ \\
\hline $\begin{array}{c}\text { Clot on varix at endoscope } \\
\text { n (\%) }\end{array}$ & $49(57)$ & $7(43.8)$ & 0.59 & $0.2-1.7$ & $\mathrm{P}=0.5$ \\
\hline $\mathrm{Hb}($ mean $\pm \mathrm{SD})$ & $7.7 \pm 2.2$ & $8.8 \pm 2.2$ & & & $\mathrm{P}=0.07$ \\
\hline $\mathrm{WBC}^{*}$ & $5.3(2.9-8.3)$ & $10.9(6.8-15.5)$ & & & $\mathrm{P}<0.0001$ \\
\hline Platelets $^{*}$ & $86(54.5-126.5)$ & $122.5(83-176.3)$ & & & $\mathrm{P}=0.06$ \\
\hline Albumin $(\mathrm{g} / \mathrm{dl})^{*}$ & $2.8 \pm 0.6$ & $2.4 \pm 0.5$ & & & $\mathrm{P}=0.005$ \\
\hline Bilirubin $(\mathrm{mg} / \mathrm{dl})^{*}$ & $1.3(0.8-2.9)$ & $8.7(1.4-19.1)$ & & & $\mathrm{P}<0.0001$ \\
\hline $\mathrm{ALT}^{*}$ & $36(22-55)$ & $75.5\left(\begin{array}{ll}37 & -147\end{array}\right)$ & & & $\mathrm{P}=0.02$ \\
\hline AST $^{*}$ & $42(27.7-64.4)$ & $116(67.5-235.8)$ & & & $\mathrm{P}<0.0001$ \\
\hline Serum creatinine ${ }^{*}$ & $0.8(0.6-1)$ & $0.9(0.6-1.4)$ & & & $\mathrm{P}=0.3$ \\
\hline Serum $\mathrm{Na}^{*}$ & $136(132.8-139)$ & $132(130.3-136)$ & & & $\mathrm{P}=0.04$ \\
\hline $\mathrm{INR}^{*}$ & $1.3(1.2-1.6)$ & $2.4(1.7-3.2)$ & & & $\mathrm{P}<0.0001$ \\
\hline CPT score & $7.5(6.8-9)$ & $11(8-11)$ & & & $\mathrm{P}<0.0001$ \\
\hline $\begin{array}{l}\text { CPT score } \\
\text { A } \\
\text { B } \\
\text { C }\end{array}$ & $\begin{array}{ll}21 & (24.4) \\
45 & (52.3) \\
20 & (23.3)\end{array}$ & $\begin{array}{c}0(0) \\
5(31.2) \\
11(68.8)\end{array}$ & & & $\mathrm{P}=0.001$ \\
\hline MELD score ${ }^{*}$ & $12.7(9.3-16.2)$ & $24.7(19.8-31)$ & & & $\mathrm{P}<0.0001$ \\
\hline Systolic blood pressure ${ }^{*}$ & $90(80-100)$ & $75(0.0-87.5)$ & & & $\mathrm{P}<0.0001$ \\
\hline
\end{tabular}

"Median (IQR). 
Table 6. Multivariate analysis of independent predictor 6 weeks mortality after acute vacireal hemorrhage.

\begin{tabular}{ccccccc}
\hline Variables & B estimate & S.E & Wald & OR & P value & $95 \%$ CI \\
\hline MELD score & 0.229 & 0.060 & 14.70 & 1.257 & $<0.0001$ & $1.12-1.41$ \\
WBCs & 0.179 & 0.079 & 5.08 & 1.196 & 0.02 & $1.02-1.40$ \\
\hline
\end{tabular}

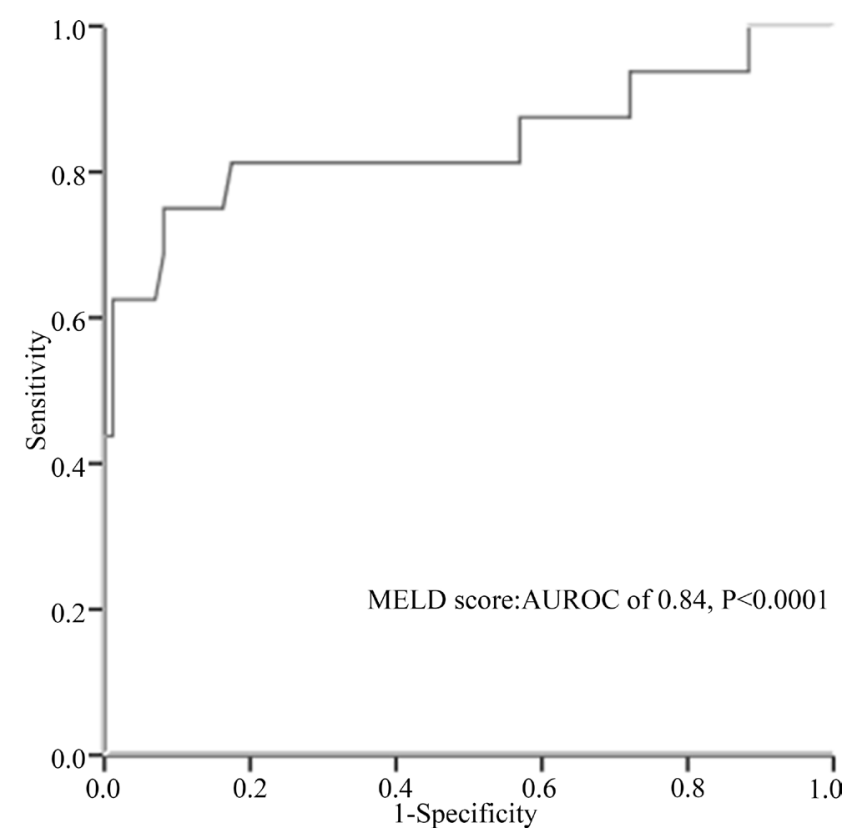

Figure 5. Receiver operating characteristic curve of model for end-stage liver disease (MELD) score in predicting 6 weeks mortality rate after variceal hemorrhage.

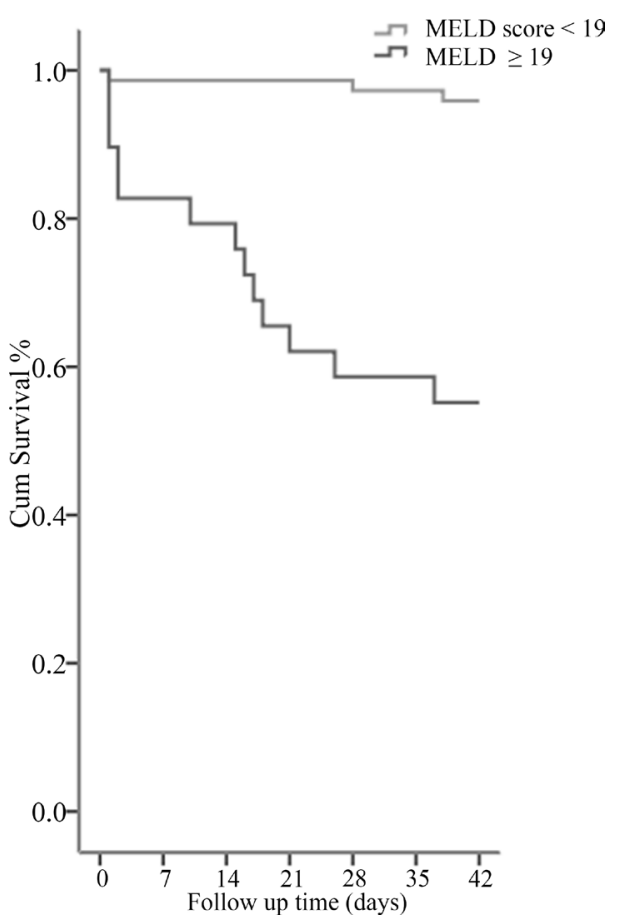

Figure 6. Kaplan-Meier estimates of 6 weeks mortality rate after acute variceal hemorrhage stratified by model for end stage liver diseases (MELD) score $(\mathrm{P}<0.001)$. 


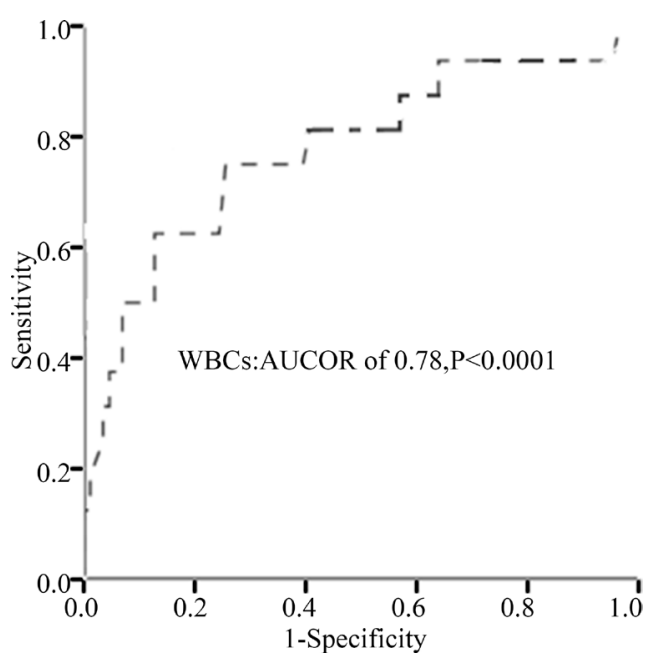

Figure 7. Receiver operating characteristic curve of WBCs in predicting 6 weeks mortality rate after variceal hemorrhage.

bleeding and mortality after AVH. Flores-Rendon et al. [25] clarified that MELD (AUROC: $0.905,95 \%$ CI: $0.801-1.00$ ) was efficacious tool for predicting in-hospital mortality related to an acute EVB episode. Kamath et al. [26] suggested that MELD was a reliable measure of short-term mortality risk in patients with end-stage liver disease of diverse etiology and severity. A study evaluating the prognostic ability of MELD in predicting mortality, Botta et al. [27] implied that MELD scoring system for both short and medium term survival was correlated with the degree of liver functional impairment. In a study of 172 cirrhotic patients with AVH and hepatocellular carcinoma, Amitrano et al. [20] stratified MELD into high risk group (MELD > 15) and low risk group (MELD $\leq 15)$, proposed that patients with MELD score $>15$ and hepatocellular carcinoma had a significantly worse survival than patients with MELD $\leq 15$ and without HCC or with early HCC either at 6 weeks or 3 months. In order to predict survival, MELD-based prognostic model was developed by Reverter et al. [6] disclosed that MELD value of $\geq 19$, predict $\geq 20 \%$ mortality compared to $<5 \%$ mortality with MELD $<11$. Bambha et al. [19] prospectively evaluated 256 cirrhotic patients in whom therapy were current standard of care for AVH including antibiotics with exclusion of hepatocellular carcinoma, pointed that patients with high MELD score ( $\geq 18$ ), 4 units of packed RBCs or more within the first 24 hours and or active bleeding at endoscope were at increase of death within 6 weeks. The mortality rate of that study were $8 \%$ and $46 \%$, for patients with MELD $<18$ compared to patients with MELD $\geq 18$, respectively. Additionally, authors of that study asserted that, MELD score of $\geq 18$ and rebleeding had increased risk of death within 6-week, whereas child class, etiology of liver diseases and aminotransferase level were not predictive of mortality. The results of our study consistent with these findings, showing 6 weeks mortality for patients with MELD $<19$ compared with patients with MELD $\geq$ 19 were $5 \%$ and $41 \%$, respectively. As in previous published reports, etiology of liver diseases, Liver enzyme and child score in the current study were not correlated with the risk of rebleeding and mortality [19] [27].

The role of MELD and HVPG in predicting mortality were evaluated in previous published studies, reported that both MELD and HVPG were independent predictors of survival. One study analyzed 101 patients with cirrhosis, stated that MELD score of $\geq 18$ and hepatic vein pressure (HVPG) $\geq 20$ were associated with 6-week mortality $(\mathrm{OR}=2.162,95 \%$ CI: $1.145-1.657, \mathrm{P}<0.001)$ and $\mathrm{OR}=1.423,95 \%$ CI: $1.222-4.084, \mathrm{P}=0.017)$ and child score were not correlated with the risk of rebleeding and mortality [19]. The prognostic ability of HVPG was assessed and compared with model based on combination of non-invasive variables in patients receiving pharmacological and endoscopic treatment, displayed that accuracy of HVPG was no better than that of prognostic ability of clinical variables based model, and that predictive models with easily and early available non-invasive objective variables from admission had enough quality in predicting survival. Moreover, HVPG measurements are not widely available and may be difficult to perform in bleeding patients. HVPG independently predicts short-term prognosis in patients with acute variceal bleeding treated with pharmacologic and endoscopic therapy, but similar predictive accuracy can be achieved using only simple clinical variables that have universal applicability [28]. Additionally, there are limitations to the generalized use of HVPG measurement [28]. First, lack of local expertise and the procedure is costly. Second, poor adherence to guidelines will ensure 
reliable and reproducible measurements. The aim of our study was to evaluate mortality within 6 weeks using non-invasive variables instead of HVPG measurement.

The other finding of present work revealed that an initial WBC over $10.3 \times 10^{9} / 1$ is independently associated with mortality within 6 weeks, similar to that finding reported previously. Predictors of mortality were evaluated prospectively by Patch et al. [29], who considered that six factors were independent prognostic risk for death: moderate to severe ascites, need for ventilation, white blood cell count, platelet count, partial thromboplastin time, and creatinine. In a recent study, an initial WBC over $10.3 \times 10^{9} / 1$ is independently associated with failure and mortality at 5 days [30]. Indeed, a more recent study realized that WBC count, creatinine level, Hb level, and prothrombin concentration were independent risk factors of mortality in ICU patients [31]. Bacterial infections are a frequent and well recognized complication of cirrhosis that may occur along the course of cirrhosis associated with gastrointestinal hemorrhage, poor liver function, low protein ascites, previous SBP, and hospitalization and adversely affect both bleeding control and mortality [11] [32]. Taking into account our data of risk patients with fever and infections (12\%), parameter such as initial elevation of WBCs could be considered as a surrogate for concomitant infections or possible infection.

\section{Conclusions}

This study demonstrated that rebleeding episodes within 5 days after acute variceal haemorrhage occurred in $14.7 \%$ of patients. 26 patients $(25.5 \%)$ suffered rebleeding within 6 weeks period. The predictive factors significantly associated with rebleeding within 6 weeks period in univariate analysis were clot on varix, serum bilirubin, CTP and MELD score. Independent risks factors remained in multivariate analysis as significant predictors of early rebleeding were clot on varix and serum bilirubin more than $3 \mathrm{mg} / \mathrm{dl}$.

Sixteen patients died (15.7\%) within 6 weeks period. Predictors of mortality with significant difference in univariate analysis were hypovolemic shock, WBCs count, serum sodium, AST, ALT, INR, bilirubin, albumin, ascites, CTP score and MELD score. By multiple regression analysis high MELD score $(>19)$ and WBC count more than $10.3 \times 10^{9} / 1$ were independent risk factors for mortality within 6 weeks period. This study may guide clinicians to be attention to patients with these risk factors.

\section{References}

[1] Graham, D.Y. and Smith, J.L. (1981) The Course of Patients after Variceal Hemorrhage. Gastroenterology, 80, 800809.

[2] de Franchis, R. (2005) Evolving Consensus in Portal Hypertension. Report of the Baveno IV Consensus Workshop on Methodology of Diagnosis and Therapy in Portal Hypertension. Journal of Hepatology, 43, 167-176. http://dx.doi.org/10.1016/j.jhep.2005.05.009

[3] Chen, W.-T., et al. (2011) MELD Score Can Predict Early Mortality in Patients with Rebleeding after Band Ligation for Variceal Bleeding. World Journal of Gasteroenterology, 17, 2120-2126. http://dx.doi.org/10.3748/wig.v17.i16.2120

[4] Garcia, T.G., Sanyal, A.J., Grace, N.D. and Carey, W. (2007) Prevention and Management of Gastroesophageal Varices and Variceal Hemorrhage in Cirrhosis. AASLD Practice Guidelines, Hepatology, 46, 922-938. http://dx.doi.org/10.1002/hep.21907

[5] D’Amico, G. and De Franchis, R. (2003) Upper Digestive Bleeding in Cirrhosis. Post-Therapeutic Outcome and Prognostic Indicators. Hepatology, 38, 599-612. http://dx.doi.org/10.1053/jhep.2003.50385

[6] Enric, R., Puneeta, T., Salvador, A. and Fanny, T. (2014) A MELD—Based Model to Determine Risk of Mortality among Patients with Acute Variceal Bleeding. Gastroenterology, 146, 412-419.

http://dx.doi.org/10.1053/j.gastro.2013.10.018

[7] Moitinho, E., Escorsell, A. and Bandi, J.C. (1999) Prognostic Value of Early Measurements of Portal Pressure in Acute Variceal Bleeding. Gastroenterology, 117, 626-631. http://dx.doi.org/10.1016/S0016-5085(99)70455-5

[8] Sarin, S.K., Kumar, A. and Angus, P.W. (2011) Asian Pacic Association for the Study of the Liver (APASL) Working Party on Portal Hypertension. Diagnosis and Management of Acute Variceal Bleeding: Asian Pacific Association for Study of the Liver Recommendations. Hepatology International, 5, 607-624. http://dx.doi.org/10.1007/s12072-010-9236-9

[9] de Franchis, R. (1996) Developing Consensus in Portal Hypertension. Journal of Hepatology, 25, 390-394. http://dx.doi.org/10.1016/S0168-8278(96)80127-9 
[10] de Franchis, R., Pascal, J., Ancona, E., Burroughs, A.K., Henderson, M., Fleig, W. and Groszmann, R. (1990) Definitions, Methodology and Therapeutic Strategies in Portal Hypertension. A Consensus Development Workshop. Journal of Hepatology, 15, 256-261.

[11] Pauwels, A., Mostefa-Kara, N., Debenes, B., Degoutte, E. and Levy, V. (1996) Systemic Antibiotic Prophylaxis after Gastrointestinal Hemorrhage in Cirrhotic Patients with a High Risk of Infection. Hepatology, 24, 802-806. http://dx.doi.org/10.1002/hep.510240408

[12] Krige, J.E., Kotze, U.K., Distiller, G., Shaw, J.M. and Bornman, P.C. (2009) Predictive Factors for Rebleeding and Death in Alcoholic Cirrhotic Patients with Acute Variceal Bleeding: A Multivariate Analysis. World Journal of Surgery, 33, 2127-2135. http://dx.doi.org/10.1007/s00268-009-0172-6

[13] Altamirano, J., Zapata, L., Augustine, S., Muntaner, L., Gonzalez, A. and Ortiz, A.L. (2008) Predicting 6 Week Mortality after Acute Variceal Bleeding: Role of Classification and Regression Tree Analysis. Annals of Hepatology, 8 , 308-315.

[14] Augustin, S., Muntaner, L. and Altamirano, J.T. (2009) Predicting Early Mortality after Acute Variceal Hemorrhage Based on Classification and Regression Tree Analysis. Clinical Gastroenterology and Hepatology, 7, 1347-1354. http://dx.doi.org/10.1016/j.cgh.2009.08.011

[15] Charif, I., Saada, K., Mellouki, I., et al. (2013) Predictors of Early Rebleeding and Mortality after Acute Variceal Haemorrhage in Patients with Cirrhosis. Open Journal of Gastroenterology, 3, 317-321. http://dx.doi.org/10.4236/ojgas.2013.37056

[16] Hunter, S.S. and Hamdy, S. (2013) Predictors of Early Re-Bleeding and Mortality after Acute Variceal Hemorrhage. Arab Journal of Gastroenterology, 14, 63-67.

[17] Zhao, J.-R., Wang, G.C., Hu, J.H. and Zhang, C.Q. (2014) Risk Factors for Early Rebleeding and Mortality in Acute Variceal Hemorrhage. World Journal of Gastroenterology, 20, 17941-17948.

[18] D'Amico, G., Garcia-Tsao, G. and Pagliaro, L. (2006) Natural History and Prognostic Indicators of Survival in Cirrhosis: A Systematic Review of 118 Studies. Journal of Hepatology, 44, 217-231. http://dx.doi.org/10.1016/j.jhep.2005.10.013

[19] Bambha, K., Kim, W.R., Pedersen, R., Bida, J.P., Kremers, W.K. and Kamath, P.S. (2008) Predictors of Early ReBleeding and Mortality after Acute Variceal Hemorrhage in Patients with Cirrhosis. Gut, 57, 814-820. http://dx.doi.org/10.1136/gut.2007.137489

[20] Amitrano, L., Guardascione, M.A., Bennato, R., Manguso, F. and Balzano, A. (2005) MELD Score and Hepatocellular Carcinoma Identify Patients at Different Risk of Short-Term Mortality among Cirrhotics Bleeding from Esophageal Varices. Journal of Hepatology, 42, 820-825. http://dx.doi.org/10.1016/j.jhep.2005.01.021

[21] Ripoll, C., Genesca, J., Araujo, I.K., Graupera, I., Augustin, S., Tejedor, M., et al. (2013) Rebleeding Prophylaxis Improves Outcomes in Patients with Hepatocellular Carcinoma: A Multicenter Case-Control Study. Hepatology, 58, 2079-2088. http://dx.doi.org/10.1002/hep.26629

[22] Gado, A., Ebeid, B., Abdelmohsen, A. and Axon, A. (2015) Predictor of Mortality in Patients with Acute Upper Gastrointestinal Hemorrhage Who Underwent Endoscopy and Confirmed to Have Variceal Hemorrhage. Alexandria Journal of Medicine, 51, 295-304. http://dx.doi.org/10.1016/j.ajme.2014.08.002

[23] Sempere, L., Palazón, J.M., Sánchez-Payá, J., Pascual, S., de Madaria, E., Poveda, J., et al. (2009) Assessing the Shortand Long-Term Prognosis of Patients with Cirrhosis and Acute Variceal Bleeding. Revista Espanola de Enfermedades Digestivas, 101, 236-248.

[24] Chen, W.T., Lin, C.Y., Sheen, I.S., et al. (2011) MELD Score Can Predict Early Mortality in Patients with Rebleeding after Band Ligation for Variceal Bleeding. World Journal of Gastroenterology, 17, 2120-2125. http://dx.doi.org/10.3748/wjg.v17.i16.2120

[25] Flores-Rendon, A.R., González-González, J.A., García-Compean, D., Maldonado-Garza, H.J. and Garza-Galindo, A.A. (2008) Model for End Stage of Liver Disease (MELD) Is Better than the Child-Pugh Score for Predicting In-Hospital Mortality Related to Esophageal Variceal Bleeding. Annals of Hepatology, 7, 230-234.

[26] Kamath, P.S., Wiesner, R.H., Malinchoc, M., et al. (2001) A Model to Predict Survival in Patients with End-Stage Liver Disease. Hepatology, 33, 464-470. http://dx.doi.org/10.1053/jhep.2001.22172

[27] Botta, F., Giannini, E., Romagnoli, P., et al. (2003) MELD Scoring System Is Useful for Predicting Prognosis in Patients with Liver Cirrhosis and Is Correlated with Residual Liver Function: A European Study. Gut, 52, 134-139. http://dx.doi.org/10.1136/gut.52.1.134

[28] Groszmann, R.J. and Wongcharatrawee, S. (2004) The Hepatic Venous Pressure Gradient: Anything Worth Doing Should Be Done Right. Hepatology, 39, 280-283. http://dx.doi.org/10.1002/hep.20062

[29] Patch, D., Nikolopoulou, V., McCormick, A., Dick, R., Armonis, A., Wannamethee, G. and Burroughs, A. (1998) Factors Related to Early Mortality after TIPS for Failed Endoscopic Therapy in Acute Variceal Bleed. Journal of He- 
patology, 28, 454-460. http://dx.doi.org/10.1016/S0168-8278(98)80320-6

[30] Amitrano, L., Guardascione, M.A., Manguso, F., et al. (2012) The Effectiveness of Current Acute Variceal Bleed Treatments in Unselected Cirrhotic Patients: Refining Short-Term Prognosis and Risk Factors. The American Journal of Gastroenterology, 107, 1872-1878. http://dx.doi.org/10.1038/ajg.2012.313

[31] Nafeh, H.M., Abdelmoneim, S.S., Hassany, S.M. and Swifee, Y.M. (2014) Risk Factors and Outcome in ICU Patients with End-Stage Liver Disease. Journal of the Arab Society for Medical Research, 9, 33-39. http://dx.doi.org/10.4103/1687-4293.137323

[32] Soares-Weiser, K., Brezis, M. and Tur-Kaspa, R. (2002) Antibiotic Prophylaxis for Cirrhotic Patients with Gastrointestinal Bleeding. Cochrane Database of Systematic Reviews, No. 2, Article No. CD002907. http://dx.doi.org/10.1002/14651858.cd002907

Submit or recommend next manuscript to SCIRP and we will provide best service for you:

Accepting pre-submission inquiries through Email, Facebook, LinkedIn, Twitter, etc.

A wide selection of journals (inclusive of 9 subjects, more than 200 journals)

Providing 24-hour high-quality service

User-friendly online submission system

Fair and swift peer-review system

Efficient typesetting and proofreading procedure

Display of the result of downloads and visits, as well as the number of cited articles

Maximum dissemination of your research work

Submit your manuscript at: http://papersubmission.scirp.org/ 